\title{
VALORES DE REFERÊNCIA DOS PARÂMETROS HEMATOLÓGICOS DE OREOCHROMIS NILOTICUS (LINAEUS, 1758) CULTIVADOS EM TANQUES-REDE EM PAULO AFONSO, NO ESTADO DA BAHIA, BRASIL.
}

\author{
AZEVEDO, T.M.P. ${ }^{1 *}$; ALBINATI, R.C.B. ${ }^{2}$; GUERRA-SANTOS, B. ${ }^{2}$; PINTO, L.F.B. ${ }^{3}$; LIRA, A.D. ${ }^{2}$; \\ MEDEIROS,S.D.C. ${ }^{2}$ \& AYRES,M.C.C. ${ }^{4}$ \\ 1. Programa Ciência Animal nos Trópicos, Escola de Veterinária, Universidade Federal da Bahia \\ (UFBA) \\ 2. Laboratório de Saúde de Organismos Aquáticos, Escola de Veterinária, UFBA \\ 3. Programa Ciência Animal nos Trópicos, Escola de Veterinária, Universidade Federal da Bahia \\ (UFBA) \\ 4. Departamento de Patologia e Patologia Clínica, Escola de Veterinária, UFBA \\ *Corresponding author: maslowa10@yahoo.com.br
}

\begin{abstract}
Azevedo, T.M.P.; Albinati, R.C.B.; Guerra-Santos, B.; Pinto, L.F.B.; Lira, A.D.; Medeiros,S.D.C. \& Ayres,M.C.C., (2016). Valores de referência dos parâmetros hematológicos de Oreochromis niloticus (Linaeus, 1758) cultivados em tanques-rede em Paulo Afonso, no Estado da Bahia, Brasil. Braz. J. Aquat. Sci. Technol. 20(2). elSSN $1983-9057$. DOI: 10.14210/bjast.v20n1. The northeastern Brazil stands out in the production of tilapia in cages and Paulo Afonso region as the fourth largest producer. Knowledge of fish physiology, the influence of management and environmental conditions is important to ensure success in cultivation. This study aimed to determine values of hematological parameters in tilapia cultured in cages in the region of Paulo Afonso, Bahia, Brazil, evaluating the effect of seasonality. There was a significant difference $(p<0.05)$ in the number of red blood cells, packed cell volume, hemoglobin concentration, mean corpuscular volume, mean corpuscular hemoglobin of concentration, the total number of leukocytes, and the relative number of lymphocytes and neutrophils. The studied haematological parameters showed influence of seasonality. The hematologic values obtained can be used in future comparative studies of fish health in similar crops.
\end{abstract}

Keywords: Hematology, Fish, Seasonality, Sanity.

\section{INTRODUÇÃO}

O sistema de criação de peixes em tanquesrede tem sua origem no sul da Ásia. Dentre as grandes vantagens na sua utilização está a possibilidade de ser praticado nos mais diferentes corpos d'água, permitir o cultivo de diferentes espécies, além de assegurar maior controle do estoque e observação dos peixes (Kubitza, 2003b/2009; Barbosa, 2009). Contemplado com características climáticas favoráveis e grande disponibilidade de água, o nordeste brasileiro destaca-se como um dos maiores polos na produção de peixes (Barbosa, 2009). O complexo de barragens de Paulo Afonso, município situado no estado da Bahia, e o quarto maior produtor do Brasil, apresenta características favoráveis e condições indispensáveis ao cultivo de peixe em tanque-rede (Souza, 2006). Diversas espécies podem ser cultivadas em tanque-rede, sendo a tilápia de grande destaque nesse sistema de cultivo.

A tilápia do Nilo (Oreochromis niloticus), de origem africana, foi introduzida no Brasil por volta dos anos 70 , tendo seu cultivo implantado em várias regiões do país (Bittencourt et al., 2003). Comercialmente, é uma das espécies de peixe que apresenta ampla aceitação nos diferentes mercados consumidores, e espaço assegurado nas exportações dos seus produtos e subprodutos (Barbosa, 2009).

Os sistemas intensivos de cultivo são caracterizados por altas densidades de estocagem, intensas práticas de manejo e condições ambientais distintas. Esses fatores induzem a excessivos níveis de estresse, desencadeando redução do crescimento, ganhos de peso, baixo desempenho reprodutivo e diminuição da resistência dos peixes a situações adversas (Iwana, 1993). Assim o conhecimento a respeito da biologia e fisiologia dos peixes é imprescindível para garantir o sucesso dos empreendimentos de cultivo (Bittencourt et al., 2003; Urbinati et al., 2004; Arana, 2004b).

Nesse contexto, tem aumentado o interesse dos pesquisadores, em caracterizar os valores dos parâmetros hematológicos e definir referências desses para avaliação do estado de saúde dos animais cultivados. A avaliação desses componentes auxilia na determinação da influência de condições fisiopatológicas que possam afetar a homeostase, contribuindo com o diagnóstico e compreensão da relação entre as características sanguíneas e saúde dos peixes e sua associação com diferentes situações ambientais (Tavares-Dias et al., 1999b, 2002ab, 2004; Seibert et al., 2001; Tavares-Dias; Moraes, 2004). 
Azevedo, T.M.P., et al. (2016). Valores de referência para hematologia de Oreochromis niloticus cultivados em tanques-rede na Bahia.

Diversos estudos sobre parâmetros sanguíneos de tilápia do Nilo têm sido revisados por pesquisadores brasileiros, mas há necessidade de se estabelecer valores de referência para populações cultivadas no Brasil. Portanto, este estudo teve como objetivo determinar valores de referência para parâmetros hematológicos em tilápias do Nilo cultivadas em tanques-rede na região de Paulo Afonso, Estado da Bahia, Brasil, bem como descrever efeitos das variáveis climáticas sazonais (sazonalidade) sobre os efeitos analisados.

\section{MATERIAL E MÉTODOS}

\section{Local de coleta dos peixes}

A pesquisa foi realizada no complexo de barragens de Moxotó e Xingó, localizados no município de Paulo Afonso, Latitude $9^{\circ}$ 24' 22"S e Longitude $38^{\circ} 12^{\prime} 53^{\prime \prime} \mathrm{W}$, no período de janeiro a dezembro de 2010, durante o verão, outono, inverno e primavera, em quatro barragens (B1, B2, B3, B4), pertencentes a Cooperativa Mista Agropecuária dos Produtores de Paulo Afonso - COMAPA e a Associação de Aquicultores de Malhada Grande. O projeto de pesquisa foi aprovado pela Comissão de Ética no Uso de Animais (CEUA-MEV/UFBA) sob o número de protocolo 13/2011, em 02/09/11.

\section{Peixes e características dos cultivos}

Foram utilizados 480 tilápias com peso e comprimento médios de $800 \mathrm{~g} \mathrm{e} 35 \mathrm{~cm}$, aparentemente saudáveis e sem lesões externas, avaliando-se 30 amostras por barragem, no verão, outono, inverno e primavera. As barragens (B1, B2, B3 e B4) apresentavam características semelhantes no arraçoamento, realizado três vezes ao dia, com ração extrusada (32\%PB), sendo uma média de 10 toneladas/ciclo/ ano. A barragem 1 possuía de 50 a 100 tanques-rede, com densidade de 100 a 300 peixes/m3 e produção de dois ciclos/ano. A barragem 2 possuía de 100 a 200 tanques-rede, com densidade de 100 peixes/m3 e produção de dois ciclos/ano. O cultivo de B1 e B2 abrange encontravam-se em uma área total de 10 ha de lâmina d'água. A barragem 3 possuía de 100 a 200 tanques-rede, com densidade de 100 a 300 peixes/ m3 e produção de um ciclo/ano. A barragem 4 possuía mais de 300 tanques-rede, com densidade de 100 a 300 peixes $/ \mathrm{m} 3$ e produção de um a três ciclos/ano, em uma área total de 80ha de lâmina d'água, semelhante a B3. Realizou-se o monitoramento dos parâmetros de qualidade da água como: temperatura, oxigênio dissolvido, $\mathrm{pH}$, amônia e condutividade elétrica, os quais foram mensurados com o uso de termômetro digital, oxímetro, phâmetro, kit colorimétrico Alfakit e condutivímetro.

\section{Colheita de sangue e procedimentos de análises}

Após despesca, 30 espécimes de tilápias do Nilo de cada barragem foram submetidos à colheita das amostras de sangue, perfazendo um total de 120 peixes. Alíquotas de $0,5 \mathrm{~mL}$ de sangue foram colhidas por punção do vaso caudal com auxílio de seringas de $3 \mathrm{~mL}$, com agulha $25 \times 7 \mathrm{~mm}$, contendo solução de ácido etilenodiaminotetraacético (EDTA10\%), e sem seguida foram transferidas para eppendorfs de $1,5 \mathrm{~mL}$ e mantidas sob refrigeração em isopor. As análises foram realizadas no Laboratório de Bioquímica Clínica de Paulo Afonso LTDA-LABIPA. Determinou-se a contagem de eritrócitos totais em câmara de Neubauer

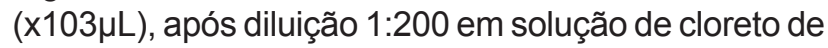
sódio (0,65\%) (Azevedo et al., 2006b); volume globular pelo método Goldenfarb et al. (1971) utilizando-se centrífuga para microhematócrito (Spin 1000) e concentração de hemoglobina determinada pelo método da cianometahemoglobina segundo Collier (1944) com leitura no espectrofotômetro (Colemann 35D). Os índices hematimétricos tais como volume corpuscular médio (VCM) e concentração da Hemoglobina Corpuscular Mediana ( $\mathrm{CHCM}$ ) foram calculados de acordo com a metodologia usual (THRALL, 2006).

Para a contagem dos leucócitos totais e diferenciais e dos trombócitos totais foram confeccionadas extensões sanguíneas coradas com May-GrunwaldGiemsa-Wrigth, utilizando o método sugerido por Tavares-dias et al. (1999; 2000). Em 10 campos homogêneos foram quantificados os leucócitos e trombócitos. De posse desses resultados e da contagem de eritrócitos previamente definidos, para cada peixe, em câmara de Neubauer, foram aplicados os seguintes cálculos:

Leucócitos $(\mu \mathrm{L})=$

Número de trombócitosx Número de eritrócitos

2000 eritrócitos

Trombócitos $(\mu \mathrm{L})=$

Número de leucócitos x Número de eritrócitos

2000 eritrócitos

\section{Análises Estatísticas}

Os dados de qualidade de água foram analisados pela ANOVA e submetidos ao teste de Tunkey com nível de $5 \%$. As demais análises não atenderam as pressuposições de normalidade, assim, optou-se por utilizar Kruskal-Wallis com nível de significância a $5 \%$. Os valores de referências foram apresentados 10 e 30 quartis, média, mediana, desvio padrão, máximo e mínimo. 


\section{RESULTADOS}

\section{Análises de água}

Os parâmetros de qualidade da água, nas barragens caracterizadas por $\mathrm{B} 1, \mathrm{~B} 2, \mathrm{~B} 3$ e B4, e os mesmos parâmetros sob o efeito das variáveis climáticas sazonais, não diferiram significativamente $(p>0,05)$, conforme demonstrados na tabela 1.

Tabela 1: Médias e desvio padrão da temperatura, oxigênio dissolvido, $\mathrm{pH}$, amônia e condutividade nas barragens da região de Paulo Afonso onde as Oreochromis niloticus foram coletadas.

\begin{tabular}{c|c|c|c|c|c|c}
\hline Barragens & $\begin{array}{c}\text { Temp } \\
\left(\mathbf{C}^{\mathbf{0}}\right)\end{array}$ & $\begin{array}{c}\mathbf{O}_{2} \\
(\mathbf{m g} / \mathbf{L})\end{array}$ & $\mathbf{p H}$ & $\begin{array}{c}\text { Amônia } \\
(\mathbf{m g} / \mathbf{L})\end{array}$ & $\begin{array}{c}\text { Condutividade } \\
(\mathbf{c m}-\mathbf{1})\end{array}$ & $\mathbf{P}$ \\
\hline B1 & $29,9 \pm 0,9$ & $7,6 \pm 1,4$ & $8,0 \pm 0,8$ & $0,1 \pm 0,1$ & $57,3 \pm 0,6$ & NS \\
B2 & $28,6 \pm 1,0$ & $7,3 \pm 0,5$ & $7,1 \pm 0,4$ & $0,1 \pm 0,1$ & $63,7 \pm 0,4$ & NS \\
B3 & $30,6 \pm 1,7$ & $6,8 \pm 0,2$ & $7,7 \pm 0,9$ & $0,1 \pm 0,1$ & $57,9 \pm 0,9$ & NS \\
B4 & $27,7 \pm 0,5$ & $7,3 \pm 0,8$ & $7,7 \pm 0,2$ & $0,1 \pm 0,1$ & $54,8 \pm 0,8$ & NS \\
Total & $29,2 \pm 1,0$ & $7,3 \pm 0,7$ & $7,6 \pm 0,6$ & $0,1 \pm 0,1$ & $58,4 \pm 0,7$ & NS \\
\hline \multicolumn{7}{l}{ NS nã }
\end{tabular}

\section{Análises Hematológicas}

Os valores de referência para número de eritrócitos, volume globular (VG), concentração de hemoglobina $(\mathrm{Hb})$, volume corpuscular médio (VCM) e concentração de hemoglobina corpuscular média (CHCM), estão apresentados na tabela 2.

Tabela 2 - Valores de referências para o eritrograma de Oreochromis niloticus, cultivadas em tanques-rede em Paulo Afonso - BA.

\begin{tabular}{lcccc}
\hline \multicolumn{1}{c}{ Variáveis } & $\mathbf{1}^{0} \mathbf{3}^{0}$ Quartil & Mediana & Média & Mínimo e Máximo \\
\hline Eritrócitos $\left(10^{6} \mu \mathrm{L}\right)$ & $1,450-2,250$ & 180 & 192,8 & $0,400-4,950$ \\
VG $(\%)$ & $35-48$ & 42 & 41,2 & $15-67$ \\
Hb (g/dL) & $9,5-12,6$ & 11,2 & 11,2 & $4,8-30,3$ \\
VCM (fL) & $162,2-301,1$ & 219,1 & 242,5 & $42,1-950$ \\
CHCM (g/dL) & $21,9-32,8$ & 27,6 & 28,7 & $10,4-98,4$ \\
\hline
\end{tabular}

As figuras de 1 a 5 apresentam os valores $10 \mathrm{e}$ 30 quartis, mediana, máximo e mínimo do número de eritrócitos, VG, Hb, VCM e CHCM das Oreochromis niloticus cultivadas em tanques-rede sob a variação sazonal. Esses parâmetros apresentaram diferença significativa $(p<0,05)$.

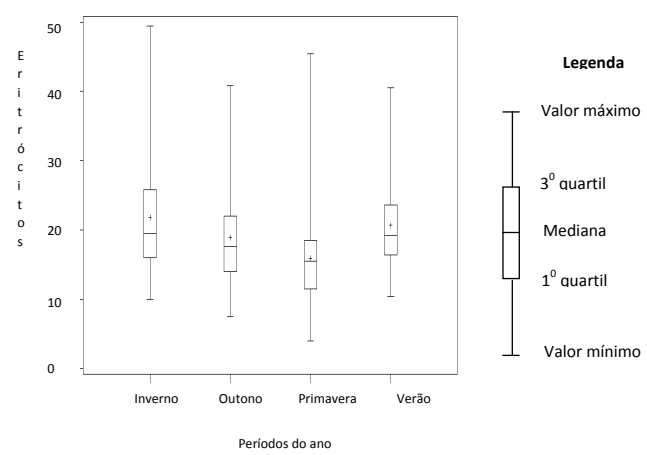

Figura 1 - Variação sazonal nos valores de referência para o número de eritrócitos $(106 \mu \mathrm{L})$ em Oreochromis niloticus, cultivadas em tanques-rede em Paulo Afonso - BA.

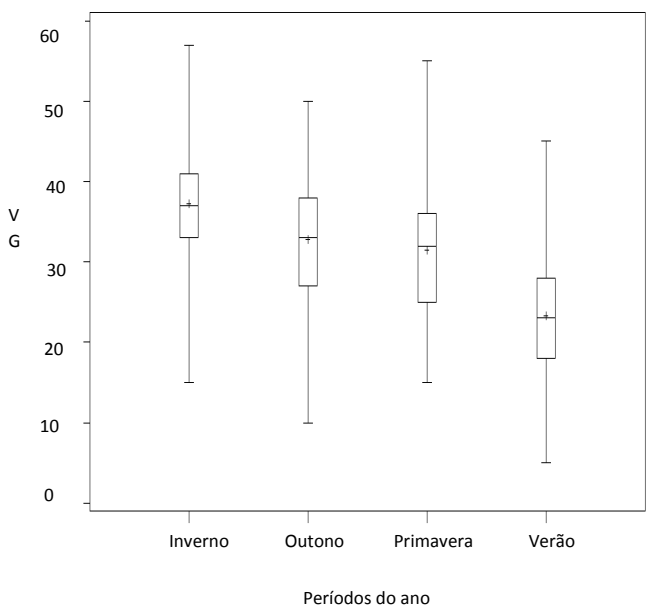

Figura 2 - Variação sazonal nos valores de referência do volume globular (\%) em Oreochromis niloticus, cultivadas em tanques-rede em Paulo Afonso - BA.

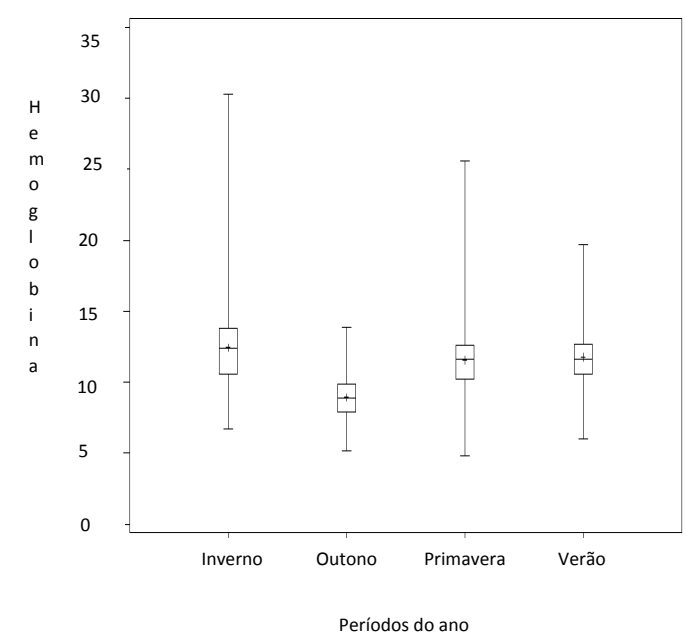

Figura 3 - Variação sazonal nos valores de referência para concentração de hemoglobina ( $\mathrm{g} / \mathrm{dL}$ ) em Oreochromis niloticus, cultivadas em tanques-rede em Paulo Afonso - BA.

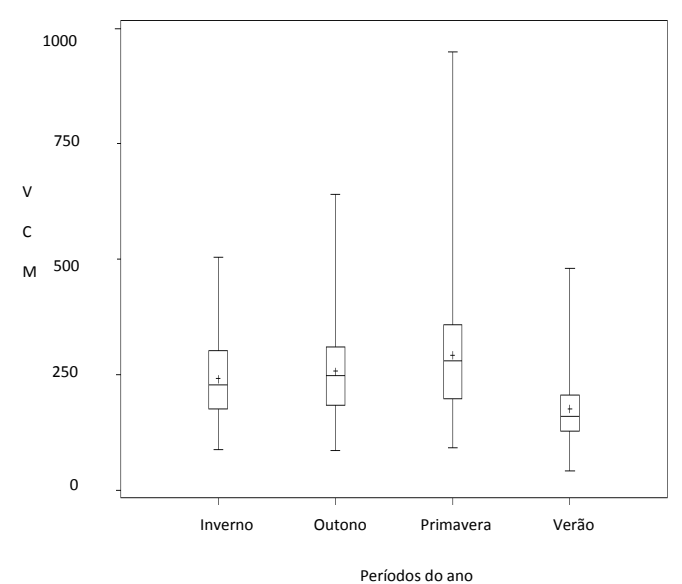

Figura 4 - Variação sazonal nos valores de referência do volume corpuscular médio (fL) em Oreochromis niloticus, cultivadas em tanques-rede em Paulo Afonso - BA. 


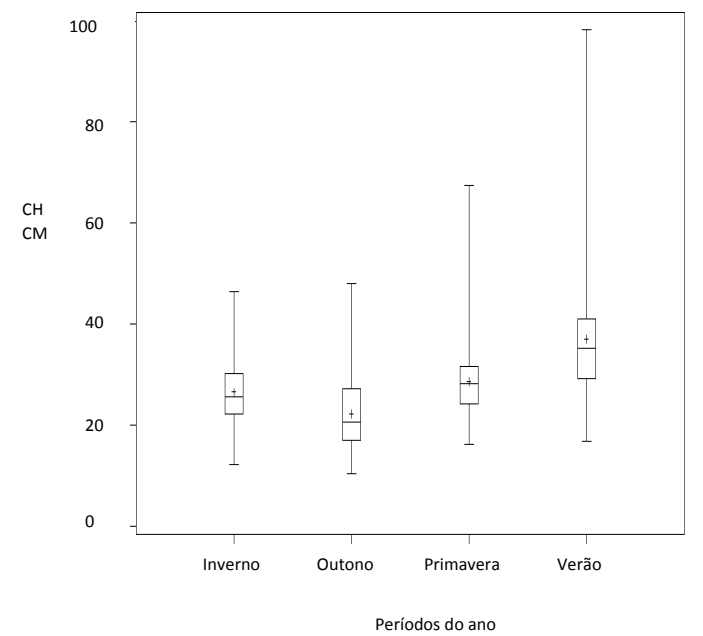

Figura 5 - Variação sazonal nos valores de referência da concentração de hemoglobina corpuscular média $(\mathrm{g} / \mathrm{dL})$ em Oreochromis niloticus, cultivadas em tanques-rede em Paulo Afonso - BA.

Os valores de referência para número de trombócitos e leucócitos são apresentados na tabela 3 . Os linfócitos foram os leucócitos predominantes em O. niloticus.

Tabela 3 - Valores de referência para trombócitos e leucócitos em Oreochromis niloticus, cultivadas em tanques-rede em Paulo Afonso - BA.

\begin{tabular}{lcccc}
\hline Parâmetros & $1^{\circ}-3^{0}$ Quartil & Mediana & Média $\pm d p$ & V.Mínimo-V.Máximo \\
\hline Trombócitos $(\mu \mathrm{L})$ & $4000-20000$ & 11000 & $12000 \pm 1000$ & $3000-20000$ \\
Leucocitos $(\mu \mathrm{L})$ & $30700-64000$ & 47000 & $48000 \pm 15000$ & $32000-67000$ \\
Linfócitos $(\mu \mathrm{L})$ & $21183-55680$ & 37130 & $38000 \pm 4000$ & $19200-62310$ \\
Linfócitos $(\%)$ & $69-87$ & 79 & $76 \pm 3,0$ & $60-93$ \\
Neutrófilos $(\mu \mathrm{L})$ & $2456-17920$ & 8930 & $9000 \pm 380$ & $1600-23450$ \\
Neutrófilos $(\%)$ & $8-28$ & 19 & $21 \pm 3,0$ & $5-35$ \\
Monócitos $(\mu \mathrm{L})$ & $0-1920$ & 470 & $300 \pm 30$ & $0-2680$ \\
Monócitos $(\%)$ & $0-3$ & 1 & $2 \pm 1$ & $0-4$ \\
\hline
\end{tabular}

As figuras de 6 a 10 apresentam os valores 10 e 30 quartis, mediana, máximo e mínimo do número de trombócitos, leucócitos, porcentagem de linfócitos (\%), neutrófilos (\%) e monócitos (\%) das Oreochromis niloticus cultivadas em tanques-rede sob a variação sazonal. Houve diferença significativa $(p<0,05)$ entre os linfócitos e neutrófilos.

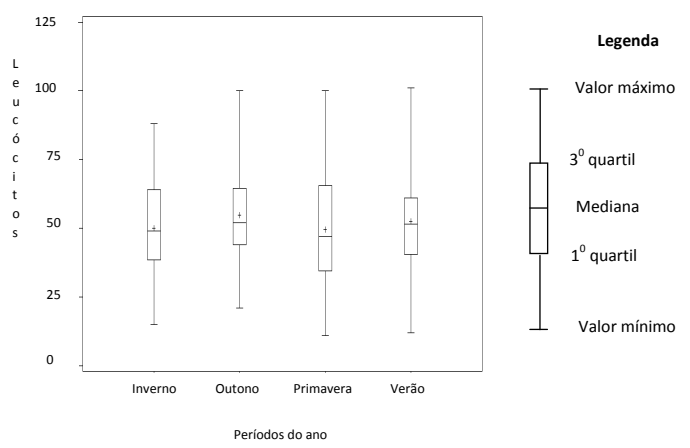

Figura 6 - Variação sazonal nos valores de referência dos leucócitos totais (x103) em Oreochromis niloticus, cultivadas em tanques-rede em Paulo Afonso - BA.

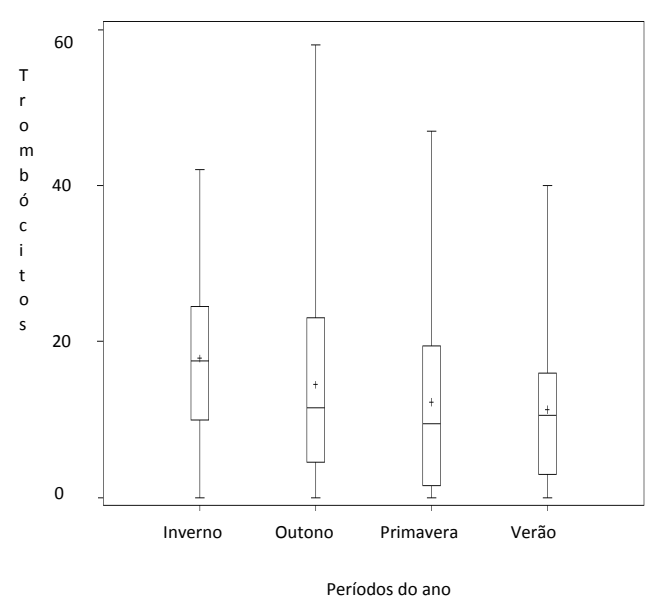

Figura 7 - Variação sazonal nos valores de referência dos trombócitos totais (x103) em Oreochromis niloticus, cultivadas em tanquesrede em Paulo Afonso - BA.

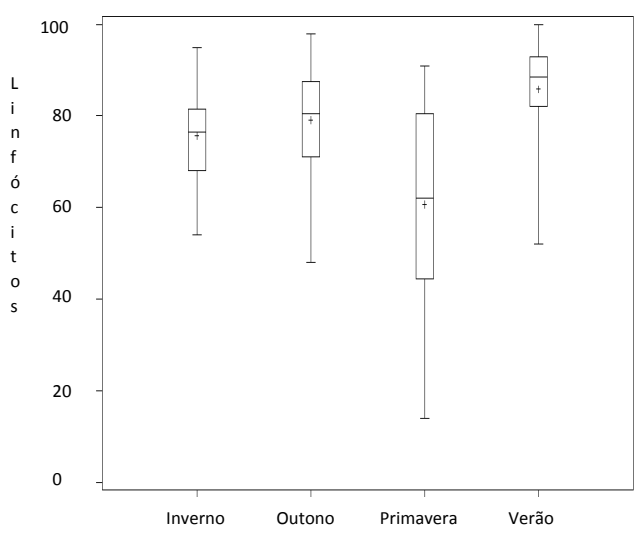

Períodos do ano

Figurā 8 - Variação sazonal nos valorés de referência do perceñtual de linfócitos (\%) em Oreochromis niloticus, cultivadas em tanquesrede em Paulo Afonso - BA.

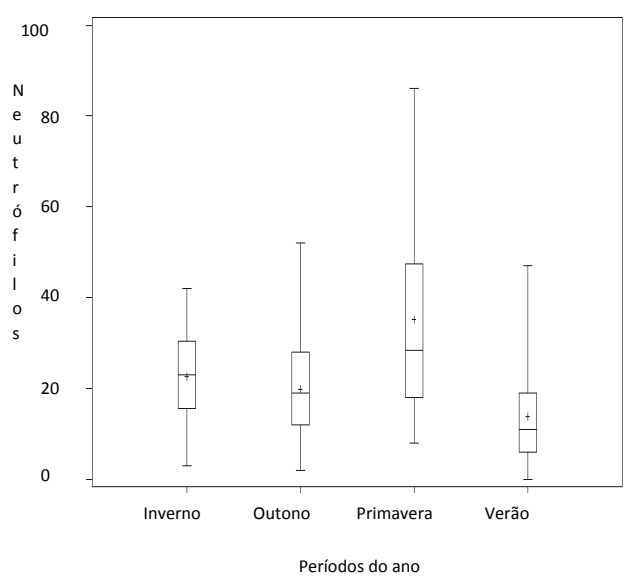

Figura 9 - Variação sazonal nos valores de referência do percentual de neutrófilos (\%) em Oreochromis niloticus, cultivadas em tanquesrede em Paulo Afonso - BA. 


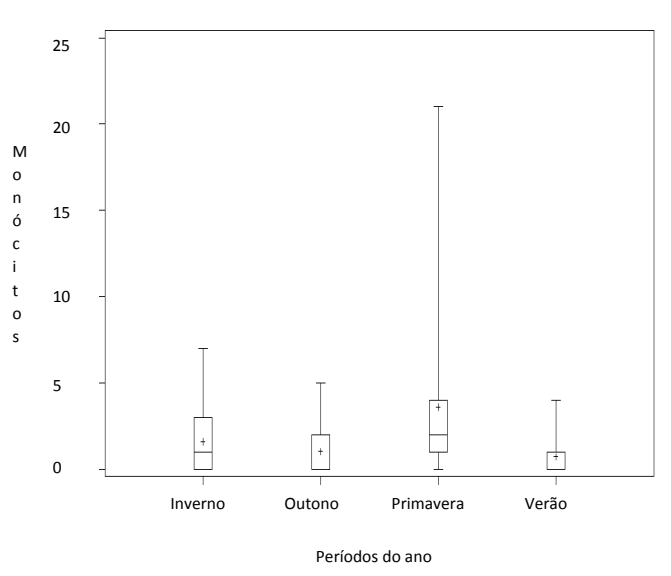

Figura 10 - Variação sazonal nos valores de referência do percentual de monócitos (\%) em Oreochromis niloticus, cultivadas em tanquesrede em Paulo Afonso - BA.

\section{DISCUSSÃO}

Os valores de temperatura, oxigênio dissolvido na água foram similares nas quatro barragens, 0 que pode estar relacionado com a similar circulação nesse reservatório. $\mathrm{O} \mathrm{pH}$, com características básicas, apresentou valores uniformes, sendo seu maior valor na barragem $1(8,0)$, provavelmente devido ao maior aporte de nutrientes. De modo geral, as concentrações de amônia não sofreram oscilações durante o estudo ( $0,1 \mathrm{mg} / \mathrm{L})$, com valores que aceitáveis para a produção de peixes em tanques-rede em reservatórios, conforme a Resolução 357/2005 do Conselho Nacional do Meio Ambiente (CONAMA). A condutividade elétrica se manteve com valores ideais ao cultivo, com maior valor na barragem 2. A condutividade elevada pode indicar poluição, o que não foi encontrado para esse estudo. Portanto, a qualidade da água apresentou valores similares aos descritos para tilápia, em outros estudos (Kubitza, 1999; 2000; Zaniboni-Filho, 2004; Boyd 1990/1998; Arana, 2004; Tavares-Dias et al., 2008) os quais foram considerados adequados para manutenção da condição de saúde dos peixes.

O número médio de eritrócitos totais 1,80 x106 foi semelhante aos valores encontrados para O niloticus por Azevedo et al. (2006) 1,5 x106 $\mu$ L; Tran-Duy et al. (2008) 1,87 x106 Nagata et al. (2009) 2,0 a 2,7 $\mathrm{x} 106 / \mu \mathrm{L}$; Ighwela et al. (2012) $4,3 \times 106$. Os valores para volume globular de $42 \%$ foram semelhantes aos relatados por Swenson (1996) 38 e $45 \%$ e de 34 a $39 \%$ registrado por Nagata et al. (2009) em O niloticus. A concentração de hemoglobina encontrada de 11,2g/ $\mathrm{dL}$ corrobora com os valores relatados para nilótica por Bittencourt et al. (2003) 10,5g/dL; Tran-Duy et al. (2008) 7,9g/dL; Nagata et al. (2009) 9,9 a 11,6g/ $\mathrm{dL}$ e Ighwela et al. (2012) 4,63mg/dL. Os valores dos índices hematométricos de VCM de 219,1fL foram superiores para os valores descritos em tilápias por Tran-Duy et al. (2008) 111fL e Nagata et al. (2009) 106fL; enquanto que os valores de $\mathrm{CHCM}$ de $27,6 \mathrm{~g} /$ $\mathrm{dL}$ foram menores ou semelhantes aos encontrados por Bittencourt et al. (2003) 35,2g/dL; Tran-Duy et al. (2008) 40g/dL; Nagata et al. (2009) 27 a 33g/dL em tilápia.

Em O. niloticus o número de eritrócitos foi maior no verão, semelhante ao que foi relatado por outros autores (Guijarro et al., 2003; Pedro et al., 2004; De Pedro et al., 2005 e Jerônimo et al., 2010). O volume globular e concentração da hemoglobina foram maiores no inverno, diferente De Pedro et al. (2004), que registraram maiores valores na primavera. 0 volume corpuscular médio e a concentração de hemoglobina corpuscular média foram maiores no verão.

Valores eritrocitários mais altos no verão podem ocorrer devido ao aumento da capacidade sanguínea em transportar o oxigênio durante períodos de demandas energéticas elevadas. Essas diferenças podem estar mais relacionadas com os hábitos e as características fisiológicas que são dependentes do ambiente no qual o peixe vive (Negrete, 2009). Montero et al. (1999), estudaram Sparus aurata observando aumento no número de eritrócitos nos meses de verão e inverno, possivelmente, pela exigência de maior aporte sanguíneo dos peixes ao maior estresse. Em contraponto, Tavares-dias (2004), observou que os pacus não apresentaram alterações em seus parâmetros eritrocitários, o que permite dizer que encontravam-se em estado de higidez. Mudanças no volume globular podem indicar hemoconcentração ou hemodiluição devido ao desbalanço osmorregulatório. Essas variações, estão relacionadas ao tamanho e atividade de cada peixe, adaptações fisiológicas das espécies, sexo, qualidade da água, fotoperíodo, dieta e época. Segundo Hrubec e Smith (2000), uma faixa de aceitação para maioria dos teleósteos seria de $20 \%$ a $45 \%$.

O aumento de eritrócitos e volume globular pode ser explicado como uma resposta ao estresse agudo. Foram encontrados maiores valores de eritrócitos no verão e inverno. As tilápias apresentaram elevados valores de VG, porém não houve interferência na homeostase. Esse aumento sugere que as tilápias se adaptaram as situações adversas de altas densidades e despesca. Segundo outros autores, fatores biológicos, fisiológicos, comportamentais e ambientais podem influenciar na variação dos parâmetros hematológicos, como a taxa de hemoglobina. Paiva et al. (2000), determinaram 7,3 a 9,7g/dL para espécies migradoras e outros autores registraram $6,6 \mathrm{~g} /$ dL e 13g/dL para outras espécies (Pitombeira, 1972; Ribeiro, 1978; Ranzani-Paiva e Godinho, 1988). Ainda nesse contexto, há correlação entre VG e Hb, e ambos 
os parâmetros estão relacionados com a atividade e habitat dos peixes, bem como, com as características nutricionais (Tavares-Dias e Moraes, 2004). $\mathrm{O}$ aumento do VCM pode representar alterações eletrolíticas e influxo de água na célula (MCdonald e Milligan, 1997) e aumento no CHCM pode ser devido a hemólise da amostra (Thrall, 2006). Neste trabalho, as amostras não apresentaram alterações nos índices hematimétricos. Concentrações mais altas de VCM e CHCM encontrados não apresentaram significado clínico importante.

O número de trombócitos encontrados de $1,1 \times 103$ foram inferiores aos valores relatados por

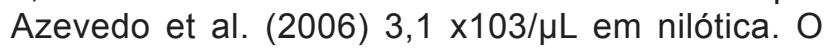
número total de leucócitos de 4,7×103 foram inferiores aos valores encontrados em tilápias por Azevedo et al. (2006) $8,2 \times 103 / \mu \mathrm{L}$; e superiores aos relatados por

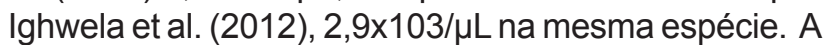
porcentagem de linfócitos de $79 \%$ foram inferiores aos relatados por Azevedo et al. 2006 96\%; e superiores aos encontrados por Ighwela et al. (2012) 52\% em tilápia. O percentual para netrófilos de $19 \%$ foram superiores aos valores encontrados em nilótica por Azevedo et al. (2006) $15 \%$ e inferiores aos valores relatados por Ighwela et al. (2012), 35\% em tilápias. O percentual de monócitos de $1 \%$ foram inferiores aos encontrados por Azevedo et al. (2006) e lghwela et al. (2012), que registraram $2 \%$ em nilótica. Eosinófilos e basófilos apresentaram baixa frequência, ou ausência, diferente dos valores relatados por Ighwela et al. (2012) $2 \%$ e $1 \%$. Os valores do eritrograma apresentaram-se de forma semelhante e diferente de outros estudos. Essas diferenças podem ser explicadas pela influência dos diversos sistemas de cultivo e da fisiologia da própria espécie nos parâmetros hematológicos.

O número de leucócitos totais foi maior no outono corroborando com os valores citados por Pedro et al. (2004) e Jerônimo et al. (2010). Por outro lado, esse estudo observou leucopenia, na primavera, enquanto que Jerônimo et al. (2010) registrou no verão. Os trombócitos foram maiores no inverno diferente dos resultados de Jerônimo et al. (2010) que observaram aumento no outono. Os linfócitos foram maiores no verão corroborando com Pedro et al. (2004) e Ranzani-Paiva et al. (2005). Em contrapartida, Hofer et al. (2000) e Jerônimo et al. (2010), registraram linfocitose no outono. Os neutrófilos se apresentaram em maior concentração na primavera diferente dos resultados encontrados por Ranzani-Paiva et al. (2004); Guiraldelli et al. (2006) e Jerônimo et al. (2010) que observaram neutrofilia no outono.

Os valores de leucócitos em teleósteos podem apresentar variações intraespecíficas influenciadas por características próprias de cada indivíduo, relacionadas ao caráter migratório dos leucócitos entre a circulação e os órgãos leucopoéticos (rim e baço), em resposta aos estímulos ambientais, (Lea master et al., 1990; Ueda et al., 1997; Tavares-Dias et al., 1999/2000; Negrete, 2009) e interespecífica como sazonalidade, reprodução, hábito alimentar, necessidades metabólicas de cada espécie (Ranzani-Paiva et al.,1999; Barros et al., 2002; Tavares-Dias e Moraes, 2004), presença de patógenos (Martins et al., 2004; Martins et al., 2008). A presença de maior número de leucócitos no sangue pode indicar melhor resposta de defesa do organismo (Tavares-Dias, 2003). Os maiores valores da contagem de leucócitos totais encontrados, possivelmente, foi devido ao estresse de densidades altas, despesca e intenso manejo.

Trombócitos são células que desempenham um papel importante no processo de coagulação do sangue, como no sistema de defesa (Matushima e Mariano, 1996; Tavares-Dias et al., 2007; Martins et al., 2008; Clauss et al., 2008; (Tavares-Dias e Oliveira, 2009). Tavares-Dias et al., (1998) descreveram redução do percentual de trombócitos em pacu infestados por Argulus sp, possivelmente, os trombócitos foram mobilizados para contribuir com os mecanismos de defesa orgânica.

Com relação aos leucócitos, foram encontradas células semelhantes a outros peixes: linfócitos, neutrófilos, monócitos em maior quantidade e baixa quantidade ou ausência de eosinófilos e basófilos. Esses resultados corroboram com valores encontrados para O. niloticus por Azevedo et al.2006. Os linfócitos desempenham um papel importante na imunidade humoral e mediada nos peixes, sendo a linfocitose sugestiva de estimulação imunogênica e a linfopenia de condições imunosupressoras (Iwama e Nakanishi, 2000; Clauss et al., 2008). Os linfócitos são os leucócitos mais encontrados na maioria dos teleósteos, o que foi comprovado com as observações deste estudo. As tilápias apresentaram maior número de linfócitos e neutrófilos circulantes o que é característico da espécie e divergindo dos estudos de Boomker, 1981 em bagre africano, na qual trombócitos e neutrófilos foram as células mais frequentes. Os eosinófilos e basófilos são escassos ou ausentes em peixes normais (Roberts, 1981; Carvalho et al., 2006), corroborando com o estudo.

Devido à intensificação dos sistemas de produção que tem como propósito alcançar índices de produtividade elevados em menor espaço físico e em menor tempo de cultivo, há necessidade de identificação dos parâmetros hematológicos e da bioquímica sanguínea de cada espécie de teleósteo em condições de normalidade para entendimento do comportamento das populações celulares em situações patológicas. O perfil hematológico pode ser utilizado para estudar o padrão normal das células sanguíneas, lesões em 
órgãos ou tecidos, infecções ou alterações ambientais devido a uma maior demanda fisiológica dos indivíduos as diversas situações. Entretanto, muitas variáveis podem influenciar significativamente nas características hematológicas: estado nutricional, sexo, fase de vida, fisiologia das espécies, manejo, condições ambientais, metodologia de colheita, anticoagulante utilizado, técnicas aplicadas e execução das análises.

\section{CONCLUSÃO}

Os parâmetros hematológicos da tilápiasdo-Nilo, cultivadas em tanques-rede, estabelecidos apresentaram influência da sazonalidade. Os valores hematológicos obtidos podem ser utilizados em outros estudos comparativos sobre a sanidade de peixes em outros cultivos semelhantes.

\section{REFERÊNCIAS BIBLIOGRÁFICAS}

Arana, L.V. Fundamentos de aqüicultura. Florianópolis: Ed. da UFSC, 2004. 348p.

Azevedo, T.M.P.; Martins, M. L.; Bozzo, F. R.; Moraes, F. R. Haematological and gills response in parasitized tilapia from valley of Tijucas River, SC, Brazil. Scientia Agricola. 63:115-120, 2006a.

Azevedo, T.M.P.; Martins, M.L.; Yamashita, M.M.; Francisco, C.J. Hematologia de Oreochromis niloticus: comparação entre peixes mantidos em piscicultura consorciada com suínos e em pesquepague no Vale do rio Tijucas, Santa Catarina, Brasil. Boletim Instituto da Pesca, São Paulo, 32(1): 41-49, 2006b.

Barbosa, A; Cultivo de tilápias em gaiolas. Sistemas de Produção, 07, Governo do Estado do Rio Grande do Norte, Natal/RN, 2009. Cimento, F.L.; Silva, E.E.V. Avaliação da qualidade da água na piscicultura em tanques-rede, Pantanal, MS. III Simpósio sobre recursos naturais e sócioeconômicos do Pantanal. Os desafios do novo milênio. 27 a 30 de 2000, Corumba-MS.

Barros, M.M., Pezzato, L.E., Kleemann, G.K., Hisano, H.; Rosa, G.J.M., 2002. Níveis de vitamina C e ferro para tilapia do Nilo (Oreochromis niloticus). Revista Brasileira de Zootecnia, v. 3, n. 6, p. 2149-2156.

Bittencourt, NL. R.; Molinari, L.M.; Scoaris, D.O.; Pedroso, R.B.; Nakamura, C.V.; Nakamura, T.U.; Filho-Abreu, B.A.; Filho-Dias, B. Haematological and biochemical values for Nile tilapia Oreochromis niloticus cultured in semi-intensive system. Acta Scientiarum. Biological Sciences Maringá, v. 25, no. 2, p. 385-389, 2003.
Bittencourt, F.; Feiden, A.; Signor, A.A.; Boscolo, W.R.; Lorenz, E.K.; Maluf, M.L.F. Densidade de estocagem e parâmetros eritrocitários de pacus criados em tanques-rede. Revista. Brasileira de Zootecnia, v.39, n.11, p.2323-2329, 2010.

Boyd, C. E. Water Quality in Ponds of Aquaculture. Alabama: Auburn University, 1990. 482p.

Boyd, C.E.; Tucker, C.S. Pond aquaculture water quality management. Boston: Kluwer Academic, 1998. 700 p.

Carvalho, G.G.P.; Pires, A.J.V.; Veloso, C.M. Silagem de resíduo de peixes em dietas para alevinos de tilápia-do-nilo Oreochromis niloticus. Revista Brasileira de Zootecnia, Viçosa, v. 35, n. 1, p. 126-130, 2006.

Clauss, T.M.; Dove, A.D.M.; Arnold, J.E. Hematologic Disorders of FishVeterinary Clinical Exotic Animal 11 (2008) 445-462.

Collier, H.B. The standardization of blood haemoglobin determinations. Canadian Medical Association. Journal, v.50, p.550-552, 1944.

De pedro, N., Guijarro, A.I., López-Patiño, M.A, Martínez-Álvarez, R., Delgado, MJ. 2005. Daily and seasonal variations in hematological and biochemical parameters in the tench, Tinca tinca Linnaeus, 1758. Aquaculture Research, v. 36, n. 12, p. $1185-1196$.

Goldenfarb, P.B.; Bowyer, F.P.; Hall, E. Reproductibility in the hematology laboratory: the microhematocrit determination. American Journal of Clinical Pathology. v.56, p.35-39, 1971.

Guijarro, A.I.; Lopez-Patiño, M.A., Pinillos, M.L. Isorna, E., De pedro, N., Alonso-Gómez, A.L., Alonsobedate, M.; Delgado, M.J. Seasonal changes in haematology and metabolic resources in the tench. Journal of Fish Biology, v. 62, n. 4, 2003, p. 803-815.

Hofer, R., Stoll, M., Romani, N., Koch, F.; Sordyl, H., 2000. Seasonal changes in blood cells of Artic char (Salvelinus alpinus L.) from a high mountain lake. Aquatic Sciences, v. 62, n. 4, p. 308-319. http:// dx.doi.org/10.1007/PL00001337

Hrubec T.C, Smith S.A. Hematology of fish. In: Feldman B.F, Zinkl JG, Jain N.C, editors. Schalm's veterinary hematology. 5th edition. Philadelphia: Lippincott Williams \& Wilkins; 2000. p. 1120-5.

Ighwela, K.A.; Ahmad, A.B.; Abol-Munaf, A.B. Haematological Changes in Nile Tilapia (Oreochromis niloticus) Fed with Varying Dietary Maltose Levels. World Journal of Fish and Marine Sciences 4 (4): 376-381, 2012.

Iwana, G. K. Intensive fish production: Course Manual UBC Access Guided Independent Study, Vancouver: The University of British Columbia, 1993, p. 130. 
Iwama, G.; Nakanishi, T. The fish immune system. Acad. Press, London, 1996, 380 p.

Kubtiza, F. Tanques-rede, rações e impacto ambiental. Revista Panorama da Aqüicultura, Rio de Janeiro, v. 9, n. 51, p. 44-50, 1999.

Kubitza, F. Qualidade da água no cultivo de peixes e camarões. Jundiaí: Fernando Kubitza, 2003b.

Jerônimo, G.T.; Lattiffe, L.V.; Speck, G.M.; Martins, M.L. Seasonal influence on the hematological parameters in cultured Nile tilapia from southern Brazil. Brazilian Journal Biology, 2011, vol. 71, no. 3, p. 719-725.

Labarrere, C.R. Perfil sanguíneo de híbridos de surubim (Pseudoplatystoma reticulatum $x$ P. coruscans) criados em diferentes densidades de estocagem. Dissertação (Mestrado) Escola de Veterinária da Universidade Federal de Minas Gerais, 2011, 63p.

Martins, M.L.; Pilarsky, F.; Onaka, E.M.; Nomura, D.T.; Fenerick jr., J.; Ribeiro, K.; Myiazaki, D.M.Y.; Castro, M.P.; Malheiros, E.B. Hematologia e resposta inflamatória aguda em Oreochromis niloticus (Osteichthyes: Cichlidae) submetida aos estímulos único e consecutivo de estresse de captura. Boletim do Instituto de Pesca, v.30, p.71-80, 2004a.

Martins, M.L.; Tavares-Dias, M.; Fujimoto, R.Y.; Onaka, E.M.; Nomura, D.T. Haematological alterations of Leporinus macrocephalus (Osteichthyes: Anostomidae) naturally infected by Goezia leporini (Nematoda: Anisakidae) in fish pond. Arquivo Brasileiro de Medicina Veterinária e Zootecnia, v.56, p.640-646, 2004b.

Martins, M.L.; Miyazaki, D.M.Y.; Moraes, F.R.; Ghiraldelli, L.; Adamante, W.B.; Mouriño, J.L. P. Ração suplementada com vitaminas $C$ e $E$ influencia a resposta inflamatória aguda em tilápia do Nilo. Vitamin $\mathrm{C}$ and $\mathrm{E}$ supplemented diet influences the acute inflammatory response in Nile tilapia. Ciência Rural, Santa Maria, v.38, n.1, p.213-218, jan-fev, 2008.

Matushima, E.R.; M. Mariano. 1996. Kinetics of the inflammatory reaction induced by carrageenin in the swinbladder of Oreochromis niloticus (Nile tilapia). Brazilia Journal Veterinary Animal. Sei. 33 (I): 5-10.

Mcdonald, G.; Milligan, C.L. 1997. Ionic, osmotic and acid-base regulation in stress. In Iwama, G.W.; Pickering, A.D.; Sumpter, J.P.; Schreck, C.B. (Eds.). Fish stress and health in aquaculture. Cambridge: University Press, p. 119-144.

Moraes, F.R.; Martins, M.L. Condições predisponentes e principais enfermidades de teleósteos em piscicultura intensiva. In: Cyrino, J.E P.; Urbinati, E.C.; Fracalossi, D.M.; Castagnolli, N. (Ed.).
Tópicos Especiais em Piscicultura de Água Doce Tropical Intensiva. São Paulo: Edit. Tecart. 2004, p.343-386.

Montero, D., Marrero, M., Izquierdo, M.S., Robaina, L., Vergara, J.M., Tort, L. (1999). Effect of vitamin E and $\mathrm{C}$ dietary supplementation on some immune parameters of gilthead seabream (Spaurus aurata) juveniles subjected to crowding stress. Aquaculture, 171: 269-278.

Nagata, M.M.; Zanuzzo, F.S.; Saita, M.V.; Biller, J. da B.; Urbinati, E.C.; Takahashi, L. S. Efeito da perseguição e exposição aérea nos parâmetros hematológicos de juvenis de tilápia-do-Nilo. V Simpósio de Ciências da UNESP e VI Encontro de Zootecnia - UNESP Dracena, 22 a 24 de setembro de 2009.

Negrete, J.C.C.; Correa, A.A.G.; Guevara, M.J.P.; Atencio garcía, V.J.; Carrasco, S.C.P. Caracterización de células sanguíneas y parámetros hematológicos en blanquillo Sorubim cuspicaudus. Zootecnia Tropical. 27(4): 393-405. 2009.

Pedro, N.; Guijarro, A.; López-Patiño, M.A.; MartínezÁlvarez, R.M.; Alonso-Bedate, M.; Delgado, M.J. Parámetros hematológicos y bioquímicos en la tenca (Tinca tinca): ritmos diarios y estacionales. Comunicación Científica CIVA 2004 (http://www. civa2004.org), 173-190.

Pitombeira, M.S. Hematologia do apaiari, Astronotus ocellatus (Cuvier, 1829) peixe teleósteo. Aspectos morfológicos e fisiológicos. 1972. Tese (Doutorado em Fisiologia) - Departamento de Fisiologia, Instituto de Biociências, Universidade Estadual de São Paulo, São Paulo, 1972.

Ranzani-Paiva, M.J.T.; Godinho, H.M., 1985. Estudos hematológicos em curimbatá. Prochilodus scrofa Steindachner, 1881 (Osteichthyes, Cypriniformes, Prochilodontidae). Série vermelha. Boletim do Instituto de Pesca, v. 12, n. 2, p. 25-35.

Ranzani-Paiva, M.J.T.; Salles, F. A.; Eiras, J.C.; Ishikawa, C.M.; Alexandrino, A. C. Análise hematológica de curimbatá (Prochilodus scrofa), pacu (Piractus mesopotamicus) e tambaqui (Colossoma macropomum) das estações de piscicultura do instituto de pesca. Boletim do Instituto de Pesca, v. 25, p. 77-83, 1999.

Ranzani-Paiva, M.J.T.; Takemoto, R.M.; Lizama, M.A.P. Sanidade de organismos aquáticos. In. RanzaniPaiva, M.J.T.; Silva-Souza, A.T., Hematologia de peixes brasileiros. São Paulo: Varela, 2004.

Roberts, R.J. 1981 Patologia de los peces, Madri, Ediciones Mundi prensa. 467p.

Sipaúba-Tavares, L.H. Limnologia Aplicada à Aqüicultura. Boletim Técnico do CAUNESP n.1, Jaboticabal: FUNEP, 1994. 70p. 
Seibert, C.S.; Guerra-Shinohara, E.M.; Carvalho, E.G.; Marques, E.E. Red blood cell parameters and osmotic fragility curve of Colossoma macropomum (Pisces, Osteichthyes, Mileinae) in captivity. Acta Scientiarum Maringá, v. 23, n. 2, p. 515-520, 2001

Souza, J. A. P. L. DE L. Estudo de impactos sociais, econômicos e ambientais, ocasionados pela piscicultura em tanques-rede na região de Paulo Afonso-Ba. Dissertação apresentada ao curso de mestrado em Ciências do Ambiente da Universidade Federal do Tocantins, em cumprimento parcial das exigências para obtenção do título de mestre em Ciências do Ambiente. Palmas, 2006.

Thrall, M. A. Hematologia e Bioquímica Clínica Veterinária. São Paulo. Roca: 2006, p.582.

Tran-Duy, A, Schrama A,J.W.; Vam dam, A.A.; Verret, J.A.J. Effects of oxygen concentration and body weight on maximum feed intake, growth and hematological parameters of Nile tilapia, Oreochromis niloticus. Aquaculture 275 (2008) 152-162.

Tavares-Dias, M. e Faustino, C.D. 1998 Parâmetros hematológicos da tilápia-do-Nilo Oreochromis niloticus (Cichlidae) em cultivo extensivo. Ars Veterinaria, Jaboticabal, 14(3): 254-263.

Tavares-Dias M.; Tenani, R.A.; Gioli, L. D.; Faustino, C.D. Características hematológicas de teleósteos brasileiros. Il. Parâmetros sangüíneos do Piaractus mesopotamicus Holmberg (Osteichthyes, Characidae) em policultivo intensivo. Revista Brasileira de Zoologia, 16 (2): 423 - 431, 1999a.

Tavares-Dias, M.; Schalch, S.H.C.; Martins, M.L.; Moraes, F.R. Características hematológicas de Oreochromis niloticus (Osteichthyes: Cichlidae) cultivadas intensivamente em "pesque-pague" do município de Franca, São Paulo, Brasil. Ars Veterinaria, Jaboticabal, 16(2): 76-82, 2000a.

Tavares-Dias, Marcos. Variáveis hematológicas de teleósteos brasileiros de importância zootécnica. Tese de doutorado - Universidade Estadual Paulista, Jaboticabal: Centro de Aqüicultura, 2003.

Tavares-Dias, M.; Moraes, F.R. Hematologia de peixes teleósteos. Ribeirão Preto: Villimpress. 2004. $144 p$.

Tavares-Dias, M; Moraes, F. R. Haematological and biochemical reference intervals for farmed channel catfish. Journal of Fish Biology. 71, 383-388, 2007.

Tavares-Dias, M., Moraes, F. R. and Martins, M. L., Hematological assessment in four Brazilian teleost fish with parasitic infections, collected in feefishing from Franca, São Paulo, Brazil. Boletim do Instituto de Pesca, v. 34, n. 2, p. 189-196, 2008.

Tavares-Dias M.; Oliveira, S. R. A review of the blood coagulation system of fish. Revista Brasileira de
Biociências. Porto Alegre, v. 7, n. 2, p. 205-224, abr./jun. 2009.

Ueda, I.K.; Egami, M.I.; Sasso, W.S.; Matushima, E.R. Estudos hematológicos em Oreochromis niloticus (Linnaeus, 1758) (Cichlidae, Teleostei) - Parte I. Brazilian Journal of Veterinary Research and Animal Science, v.34, p.270-275, 1997.

Urbinati, E.C.; Abreu, J.S.; Camargo, A.C.S.; Landines, M.A. Loading and transport stress in juvenile matrinxã (Brycon cephalus) at various densities. Aquaculture. v.229, p.389-400, 2004.

Zaniboni filho, E. Piscicultura das espécies exóticas de água doce In Poli, CR., Poli, ATB. Andreatta, ER. and Beltrame, E. (Eds) Aqüicultura: experiências brasileiras. Florianópolis. p. 309-336, 2004.

Wintrobe, M.M. 1934. Variations on the size and hemoglobin content of erythrocytes in the blood various vertebrates. Fol. Haematol. 5:32-49.

Brasil. Boletim Instituto da Pesca, São Paulo, 32(1): 41-49, 2006b.

Barbosa, A; Cultivo de tilápias em gaiolas. Sistemas de Produção, 07, Governo do Estado do Rio Grande do Norte, Natal/RN, 2009.

Barbosa, D.S.; Oliveira, M.D. DE; Nascimento, F.L.; Silva, E.E.V. Avaliação da qualidade da água na piscicultura em tanques-rede, Pantanal, MS. III Simpósio sobre recursos naturais e sócioeconômicos do Pantanal. Os desafios do novo milênio. 27 a 30 de 2000, Corumba-MS.

Barros, M.M., Pezzato, L.E., Kleemann, G.K., Hisano, H.; Rosa, G.J.M., 2002. Níveis de vitamina C e ferro para tilapia do Nilo (Oreochromis niloticus). Revista Brasileira de Zootecnia, v. 3, n. 6, p. 2149-2156.

Bittencourt, NL. R.; Molinari, L.M.; Scoaris, D.O.; Pedroso, R.B.; Nakamura, C.V.; Nakamura, T.U.; Filho-Abreu, B.A.; Filho-Dias, B. Haematological and biochemical values for Nile tilapia Oreochromis niloticus cultured in semi-intensive system. Acta Scientiarum. Biological Sciences Maringá, v. 25, no. 2, p. 385-389, 2003.

Bittencourt, F.; Feiden, A.; Signor, A.A.; Boscolo, W.R.; Lorenz, E.K.; Maluf, M.L.F. Densidade de estocagem e parâmetros eritrocitários de pacus criados em tanques-rede. Revista. Brasileira de Zootecnia, v.39, n.11, p.2323-2329, 2010.

Boyd, C. E. Water Quality in Ponds of Aquaculture. Alabama: Auburn University, 1990. 482p.

Boyd, C.E.; Tucker, C.S. Pond aquaculture water quality management. Boston: Kluwer Academic, 1998. 700 p.

Carvalho, G.G.P.; Pires, A.J.V.; Veloso, C.M. Silagem de resíduo de peixes em dietas para alevinos de tilápia-do-nilo Oreochromis niloticus. Revista Brasileira de Zootecnia, Viçosa, v. 35, n. 1, p. 
126-130, 2006.

Clauss, T.M.; Dove, A.D.M.; Arnold, J.E. Hematologic Disorders of Fish Veterinary Clinical Exotic Animal 11 (2008) 445-462.

Collier, H.B.The standardization of blood haemoglobin determinations. Canadian Medical Association. Journal, v.50, p.550-552, 1944.

De pedro, N., Guijarro, A.I., López-Patiño, M.A, Martínez-Álvarez, R., Delgado, MJ. 2005. Daily and seasonal variations in hematological and biochemical parameters in the tench, Tincatinca Linnaeus, 1758. Aquaculture Research, v. 36, n. 12, p. 1185-1196.

Goldenfarb, P.B.; Bowyer, F.P.; Hall, E. Reproductibility in the hematology laboratory: the microhematocrit determination. American Journal of Clinical Pathology. v.56, p.35-39, 1971.

Guijarro, A.I.; Lopez-Patiño, M.A., Pinillos, M.L. Isorna, E., De pedro, N., Alonso-Gómez, A.L., Alonsobedate, M.; Delgado, M.J. Seasonal changes in haematology and metabolic resources in the tench. Journal of Fish Biology, v. 62, n. 4, 2003, p. 803-815.

Hofer, R., Stoll, M., Romani, N., Koch, F.; Sordyl, H., 2000. Seasonal changes in blood cells of Artic char (Salvelinus alpinus L.) from a high mountain lake. Aquatic Sciences, v. 62, n. 4, p. 308-319. http:// dx.doi.org/10.1007/PL00001337

Hrubec T.C, Smith S.A. Hematology of fish. In: Feldman B.F, Zinkl JG, Jain N.C, editors. Schalm's veterinary hematology. 5th edition. Philadelphia: Lippincott Williams \& Wilkins; 2000. p. 1120-5.

Ighwela, K.A.; Ahmad, A.B.; Abol-Munaf, A.B. Haematological Changes in Nile Tilapia (Oreochromis niloticus) Fed with Varying Dietary Maltose Levels. World Journal of Fish and Marine Sciences 4 (4): 376-381, 2012.

Iwana, G. K. Intensive fish production: Course Manual UBC Access Guided Independent Study, Vancouver: The University of British Columbia, 1993, p. 130.

Iwama, G.; Nakanishi, T. The fish immune system. Acad. Press, London, 1996, 380 p.

Kubtiza, F. Tanques-rede, rações e impacto ambiental. Revista Panorama da Aqüicultura, Rio de Janeiro, v. 9, n. 51, p. 44-50, 1999.

Kubitza, F. Qualidade da água no cultivo de peixes e camarões. Jundiaí: Fernando Kubitza, 2003b.

Jerônimo, G.T.; Lattiffe, L.V.; Speck, G.M.; Martins, M.L. Seasonal influence on the hematological parameters in cultured Nile tilapia from southern Brazil. Brazilian Journal Biology, 2011, vol. 71, no. 3, p. 719-725.

Labarrere, C.R. Perfil sanguíneo de híbridos de surubim (Pseudoplatystoma reticulatum $\times \mathrm{P}$. coruscans) criados em diferentes densidades de estocagem. Dissertação (Mestrado) Escola de Veterinária da Universidade Federal de Minas Gerais, 2011, 63p.

Martins, M.L.; Pilarsky, F.; Onaka, E.M.; Nomura, D.T.; Fenerick jr., J.; Ribeiro, K.; Myiazaki, D.M.Y.; Castro, M.P.; Malheiros, E.B. Hematologia e resposta inflamatória aguda em Oreochromis niloticus (Osteichthyes: Cichlidae) submetida aos estímulos único e consecutivo de estresse de captura. Boletim do Instituto de Pesca, v.30, p.71-80, 2004a.

Martins, M.L.; Tavares-Dias, M.; Fujimoto, R.Y.; Onaka, E.M.; Nomura, D.T. Haematological alterations of Leporinus macrocephalus (Osteichthyes: Anostomidae) naturally infected by Goezia leporini (Nematoda: Anisakidae) in fishpond. Arquivo Brasileiro de Medicina Veterinária e Zootecnia, v.56, p.640-646, 2004b.

Martins, M.L.; Miyazaki, D.M.Y.; Moraes, F.R.; Ghiraldelli, L.; Adamante, W.B.; Mouriño, J.L. P. Ração suplementada com vitaminas C e E influencia a resposta inflamatória aguda em tilápia do Nilo. Vitamin C and E supplemented diet influences the acute inflammatory response in Nile tilapia.Ciência Rural, Santa Maria, v.38, n.1, p.213-218, jan-fev, 2008.

Matushima, E.R.; M. Mariano. 1996. Kinetics of the inflammatory reaction induced by carrageen in in the swin bladder of Oreochromis niloticus (Nile tilapia). Brazilia Journal Veterinary Animal. Sei. 33 (I): 5-10.

Mcdonald, G.; Milligan, C.L. 1997. Ionic, osmotic and acid-base regulation in stress. In Iwama, G.W.; Pickering, A.D.; Sumpter, J.P.; Schreck, C.B. (Eds.). Fish stress and health in aquaculture. Cambridge: University Press, p. 119-144.

Moraes, F.R.; Martins, M.L. Condições predisponentes e principais enfermidades de teleósteos em piscicultura intensiva. In: Cyrino, J.E P.; Urbinati, E.C.; Fracalossi, D.M.; Castagnolli, N. (Ed.). Tópicos Especiais em Piscicultura de Água Doce Tropical Intensiva.São Paulo: Edit. Tecart. 2004, p.343-386.

Montero, D., Marrero, M., Izquierdo, M.S., Robaina, L., Vergara, J.M., Tort, L. (1999). Effect of vitamin E and $\mathrm{C}$ dietary supplementation on some immune parameters of gilthead seabream (Spaurus aurata) juveniles subjected to crowding stress. Aquaculture, 171: 269-278.

Nagata, M.M.; Zanuzzo, F.S.; Saita, M.V.; Biller, J. da B.; Urbinati, E.C.; Takahashi, L. S. Efeito da perseguição e exposição aérea nos parâmetros hematológicos de juvenis de tilápia-do-Nilo. V Simpósio de Ciências da UNESP e VI Encontro de 
Zootecnia - UNESP Dracena, 22 a 24 de setembro de 2009.

Negrete, J.C.C.; Correa,A.A.G.; Guevara, M.J.P.;Atencio garcía, V.J.; Carrasco, S.C.P. Caracterización de células sanguíneas y parámetros hematológicos enblanquillo Sorubimcus picaudus. Zootecnia Tropical. 27(4): 393-405. 2009.

Pedro, N.; Guijarro, A.; López-Patiño, M.A.; MartínezÁlvarez, R.M.; Alonso-Bedate, M.; Delgado, M.J. Parámetros hematológicos y bioquímicos enlatenca (Tincatinca): ritmos diarios y estacionales. Comunicación Científica CIVA 2004 (http://www. civa2004.org), 173-190.

Pitombeira, M.S. Hematologia do apaiari, Astronotus ocellatus (Cuvier, 1829) peixe teleósteo. Aspectos morfológicos e fisiológicos. 1972. Tese (Doutorado em Fisiologia) - Departamento de Fisiologia, Instituto de Biociências, Universidade Estadual de São Paulo, São Paulo, 1972.

Ranzani-Paiva, M.J.T.; Godinho, H.M., 1985. Estudos hematológicos em curimbatá. Prochilodus scrofa Steindachner, 1881 (Osteichthyes, Cypriniformes, Prochilodontidae). Série vermelha. Boletim do Instituto de Pesca, v. 12, n. 2, p. 25-35.

Ranzani-Paiva, M.J.T.; Salles, F. A.; Eiras, J.C.; Ishikawa, C.M.; Alexandrino, A. C. Análise hematológica de curimbatá (Prochilodusscrofa), pacu (Piractus mesopotamicus) e tambaqui (Colossoma macropomum) das estações de piscicultura do instituto de pesca. Boletim do Instituto de Pesca, v. 25, p. 77-83, 1999.

Ranzani-Paiva, M.J.T.; Takemoto, R.M.; Lizama, M.A.P. Sanidade de organismos aquáticos. In. RanzaniPaiva, M.J.T.; Silva-Souza, A.T., Hematologia de peixes brasileiros. São Paulo: Varela, 2004.

Roberts, R.J. 1981 Patologia de lospeces, Madri, EdicionesMundi prensa. 467p.

Sipaúba-Tavares, L.H. Limnologia Aplicada à Aqüicultura. Boletim Técnico do CAUNESP n.1, Jaboticabal:FUNEP, 1994. 70p.

Seibert, C.S.; Guerra-Shinohara, E.M.; Carvalho, E.G.; Marques, E.E. Red blood cell parameter sandos motic fragility curve of Colossoma macropomum (Pisces, Osteichthyes, Mileinae) in captivity. Acta Scientiarum Maringá, v. 23, n. 2, p. 515-520, 2001

Souza, J. A. P. L. de L. Estudo de impactos sociais, econômicos e ambientais, ocasionados pela piscicultura em tanques-rede na região de Paulo Afonso-Ba. Dissertação apresentada ao curso de mestrado em Ciências do Ambiente da Universidade Federal do Tocantins, em cumprimento parcial das exigências para obtenção do título de mestre em Ciências do Ambiente. Palmas, 2006.

Thrall, M. A. Hematologia e Bioquímica Clínica
Veterinária. São Paulo. Roca:2006, p.582.

Tran-Duy, A, Schrama A,J.W.; Vam dam, A.A.; Verret, J.A.J. Effects of oxygen concentration and body weight on maximum feed intake, growth and hematological parameters of Nile tilapia, Oreochromis niloticus. Aquaculture 275 (2008) 152-162.

Tavares-Dias, M. e Faustino, C.D. 1998 Parâmetros hematológicos da tilápia-do-Nilo Oreochromis niloticus (Cichlidae) em cultivo extensivo. Ars Veterinaria, Jaboticabal, 14(3): 254-263.

Tavares-Dias M.; Tenani, R.A.; Gioli, L. D.; Faustino, C.D. Características hematológicas de teleósteos brasileiros. II. Parâmetros sangüíneos do Piaractus mesopotamicus Holmberg (Osteichthyes, Characidae) em policultivo intensivo. Revista Brasileira de Zoologia, 16 (2): 423 - 431, 1999a.

Tavares-Dias, M.; Schalch, S.H.C.; Martins, M.L.; Moraes, F.R. Características hematológicas de Oreochromis niloticus (Osteichthyes: Cichlidae) cultivadas intensivamente em "pesque-pague" do município de Franca, São Paulo, Brasil. Ars Veterinaria, Jaboticabal, 16(2): 76-82, 2000a.

Tavares-Dias, Marcos. Variáveis hematológicas de teleósteos brasileiros de importância zootécnica. Tese de doutorado - Universidade Estadual Paulista, Jaboticabal: Centro de Aqüicultura, 2003.

Tavares-Dias, M.; Moraes, F.R. Hematologia de peixes teleósteos. Ribeirão Preto: Villimpress. 2004. $144 p$.

Tavares-Dias, M; Moraes, F. R. Haematological and biochemical reference intervals for farmed channel catfish. Journal of Fish Biology. 71, 383-388, 2007.

Tavares-Dias, M., Moraes, F. R. and Martins, M. L., Hematological assessment in four Brazilian teleost fish with parasitic infections, collected in feefishing from Franca, São Paulo, Brazil. Boletim do Instituto de Pesca, v. 34, n. 2, p. 189-196, 2008.

Tavares-Dias M.; Oliveira, S. R. A review of the blood coagulation system of fish. Revista Brasileira de Biociências. Porto Alegre, v. 7, n. 2, p. 205-224, abr./jun. 2009.

Ueda, I.K.; Egami, M.I.; Sasso, W.S.; Matushima, E.R. Estudos hematológicos em Oreochromis niloticus (Linnaeus, 1758) (Cichlidae, Teleostei) - Parte I. Brazilian Journal of Veterinary Researchand Animal Science, v.34, p.270-275, 1997.

Urbinati, E.C.; Abreu, J.S.; Camargo, A.C.S.; Landines, M.A. Loading and transport stress in juvenile matrinxã (Bryconcephalus) at various densities. Aquaculture. v.229, p.389-400, 2004.

Zaniboni filho, E. Piscicultura das espécies exóticas de água doce In Poli, CR., Poli, ATB. Andreatta, ER. and Beltrame, E. (Eds) Aqüicultura: experiências brasileiras. Florianópolis. p. 309-336, 2004. 
Azevedo, T.M.P., et al. (2016). Valores de referência para hematologia de Oreochromis niloticus cultivados em tanques-rede na Bahia.

Wintrobe, M.M. 1934. Variations on the size and hemoglobin content of erythrocytes in the blood various vertebrates. Fol. Haematol. 5:32-49.

Submetido: Junho/2013

Revisado: Outubro/2015

Aceito: Fevereiro/2016 